\title{
A Novel High-sensitivity ADS-B Receiver Based on RF Direct Logarithmic Detecting
}

\author{
Wei Peng, Yubai Li \\ DSP Lab, School of Communication and Information \\ Engineering \\ University of Electronic Science and Technology of \\ China \\ ChengDu, China \\ scootpeng@hotmail.com
}

\author{
Huan Li,Bolong Wen \\ DSP Lab, School of Communication and Information \\ Engineering \\ University of Electronic Science and Technology of \\ China \\ ChengDu, China \\ photon_lee@126.com
}

\begin{abstract}
ADS-B will significantly increase the availability and quality of air traffic surveillance of future air traffic management systems. The circuit design of traditional ADS-B receiver based on superheterodyne receiving is complex but the sensitivity is not so satisfactory. This paper proposes a novel high-sensitivity ADS-B receiver, which is totally different from the traditional superheterodyne ADS-B receiver. The sensitivity, noise figure, spurious-free dynamic range and bit error rate of the receiver are analyzed in this paper. Finally, the performance of the receiver is tested by both signals from analog signal generator and signals from real world.
\end{abstract}

Keywords- ADS-B Receiver, direct logarithmic detecting, high sensitivity

\section{INTRODUCTION}

Automatic Dependent Surveillance-Broadcast (ADS-B) [1] will be a key element of future air traffic management systems, such as the Single European Sky ATM Research program (SESAR) and the United States' NextGen program. Australia is the first country with full, nationwide ADS-B coverage. The United States will require the majority of aircraft operating within its airspace to be equipped with some form of ADS-B Out by January 1, 2020 [2].

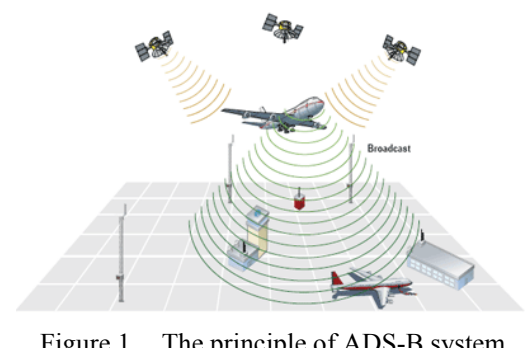

Fig. 1 shows the principle of an ADS-B system. Unlike the current surveillance technique, where a ground based radar transmits interrogating signals and uses the replies from aircraft transponders to determine the locations of the aircrafts [3], ADS-B equipped aircraft will broadcast their Global Navigation Satellite System (GNSS) positions once per second. The information received by air traffic controllers, and other ADS-B aircraft, includes the aircraft's identification, altitude, speed, velocity, projected path and other useful information [4].

Because receive sensitivity indicates how faint an input signal can be to be successfully received by the receiver, the lower power level, the better. ADS-B receiver sensitivity, which is focused on the RF receiver module, is now the key factor of the whole system. This paper proposes a novel high sensitive ADS-B receiver based on RF direct logarithmic detecting, and it is verified by both theoretical analysis and practical test. We can conclude that the performance of the receiver is satisfactory.

\section{TRADITIONAL ADS-B RECEIVER}

The current art for receiving the ADS-B signal is based on superheterodyne receiving[5], and the block diagram of this receiver is shown in figure 2 .

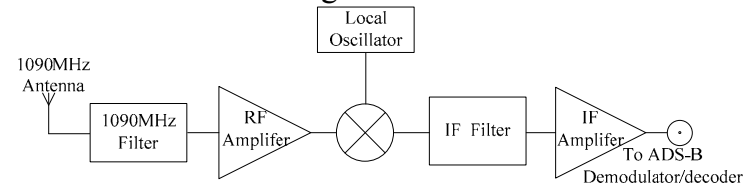

Figure 2. Block diagram of a typical ADS-B receiver

Fig. 2 shows the minimum requirements for a traditional ADS-B receiver design. The following essential elements are common to all this sort of circuits: a $1090 \mathrm{MHz}$ receiving antenna, a tuned stage which may optionally contain amplification (RF amplifier), a variable frequency local oscillator (LO), a frequency mixer, a band pass filter, intermediate frequency (IF) amplifier, and a demodulator plus additional circuitry to amplify or process the original ADS-B signal.The traditional ADS-B receiver needs a lot of modules such as a low noise RF/IF amplifier, two high Quality RF filters, a phase locked loop (PLL), a voltage control oscillator (VCO) and a IF filter, such that the circuit design is very complex and the sensitivity is not so satisfactory.

\section{Description ABOUt THE High Sensitivity ADS-B RECEIVER}

In this paper, a novel ADS-B receiver is introduced. This receiver adopts direct logarithmic detecting from RF signal. 
The circuit is simple but the sensitivity is high. This receiver is verified by both theoretical analysis and practical test.

Figure 3 shows the block diagram of this high sensitivity ADS-B receiver.

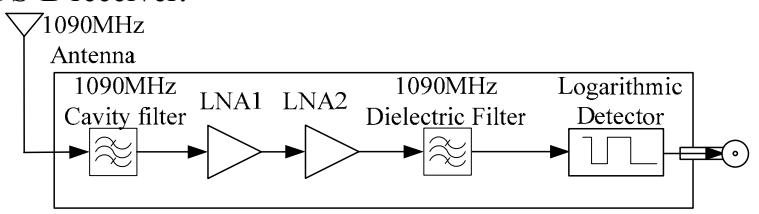

Figure 3. Block diagram of high sensitivity ADS-B receiver

In this receiver, the gain of the antenna is $10 \mathrm{~dB}$, which is used to counteract with the loss of the Cables by increasing the received signal strength.

The first RF filter is a cavity filter whose insertion loss is $1.5 \mathrm{~dB}$, fluctuation is smaller than $0.5 \mathrm{~dB}$ in passband and it will loss $3 \mathrm{~dB}$ power at $1090 \mathrm{MHz} \pm 7.5 \mathrm{MHz}$.

The filtered ADS-B signal is fed into two LNAs. The characters of the first LNA is $14 \mathrm{~dB}$ gain, $0.9 \mathrm{~dB}$ noise figure and $15 \mathrm{dBm}$ output OIP3 .The characters of the second LNA is $22 \mathrm{~dB}$ gain, $1.5 \mathrm{~dB}$ noise figure and $25 \mathrm{dBm}$ output OIP3.

The amplified signal is sent to a secondary dielectric filter whose insertion loss is $5 \mathrm{~dB}$ and it will loss $3 \mathrm{~dB}$ power at $1090 \mathrm{MHz} \pm 6 \mathrm{MHz}$ and loss more than $40 \mathrm{~dB}$ power at $1090 \mathrm{MHz} \pm 25 \mathrm{MHz}$.

Since the receiver adopts direct logarithmic detecting for $\mathrm{RF}$ signal, the output of the detector is baseband ADS-B signal.

\section{THEORETICAL ANALYSIS OF THE RECEIVER}

Sensitivity in a receiver is defined as the minimum input signal Pin,min required to produce a specified SNR at the output port of the receiver and is defined as the mean noise power at the input port of the receiver times the minimum required SNR at the output of the receiver [6].

$$
P_{i n, \min }=\frac{P_{o u t, \min }}{G p}=\frac{N_{o}}{G p} \frac{P_{o u t, \min }}{N_{o}}
$$

Where Pout,min is the required minimum output power after the signal amplifying, N0 is the total noise power of the whole system.

$$
N_{o}=k B\left[T_{a}+(F-1) T_{o}\right] G_{p}
$$

Besides, the relationship between Pout,min and $S N R_{o, \min }$ can by expressed as follow:

$$
S N R_{o, \min }=\frac{P_{o u t, \min }}{N_{o}}
$$

So the sensitivity Pin,min can be re-written as :

$$
P_{i n, \text { min }}=k B\left[T_{a}+(F-1) T_{o}\right] S N R_{o, \text { min }}
$$

Where $\mathrm{k}$ is Boltzmann constant, $\mathrm{T} 0$ is the room temperature, $\mathrm{B}$ is the equivalent noise bandwidth and SNR could be considered as a constant.

$\mathrm{NF}$ is the Noise figure of whole system, and it is defined as follow [8]:

$$
N F=10 \log \left(\frac{S N R_{\text {in }}}{S N R_{\text {out }}}\right)=S N R_{\text {in }, d B}-S N R_{\text {out }, d B}
$$

Where SNRin and SNRout are the input and output power signal-to-noise ratios. SNRin, $\mathrm{dB}$ and SNRout,dB are measured in decibels $(\mathrm{dB})$.

If several devices are cascaded, the total noise factor can be calculated by Friis' Formula:

$$
N F=N F_{1}+\frac{N F_{2}-1}{G_{1}}+\frac{N F_{3}-1}{G_{1} G_{2}}+\ldots \ldots+\frac{N F_{n}-1}{G_{1} G_{2} \ldots G_{n-1}}
$$

Where Fn is the noise factor for the $\mathrm{n}$-th device and $\mathrm{Gn}$ is the power gain (linear, not in $\mathrm{dB}$ ) of the $n$-th device. In a well designed receive chain, only the noise factor of the first amplifier should be significant.

From formula (7), it is concluded that the Noise figure mainly depend upon the first and secondary filter. $\mathrm{K}$ and $\mathrm{T} 0$ could be considered as a constant, such that the sensitivity of the system can be simplified as:

$P_{i n, \text { min }}(\mathrm{dBm})=-174(\mathrm{dBm} / \mathrm{Hz})+10 \log B+N F+S N R_{\text {min }}$

In this system, the bandwidth of the first RF filter is 15 $\mathrm{MHz}$, the NF of the whole system is $5 \mathrm{~dB}$ from testing result. The minimum SNR needed in baseband signal processing is $8 \mathrm{~dB}$, such that we can conclude that the sensitivity of the receiver is:

$$
P_{i n, \min }(\mathrm{dBm})=-174+10 \log 15 \times 10^{6}+5+8=-90 \mathrm{dBm}
$$

This result is consistent with the practical test.

Besides of the sensitivity, the Spurious-Free Dynamic Range (SFDR) is another important index to the receiver. According to the classic formula calculating the dynamic range [7]:

$$
\begin{aligned}
& D R_{f}=\frac{P_{i n, \text { max }}}{P_{i n, \text { min }}} \\
& P_{i n, \text { min }}=\mathrm{F}_{\mathrm{t}}+\mathrm{SNR}_{\text {min }}, P_{i n, \text { max }}=\frac{2 \mathrm{P}_{\mathrm{IIP} 3}+\mathrm{F}_{\mathrm{t}}}{3} \\
& D R_{f}(d B)=\frac{2}{3}\left[P_{I I P_{3}}(d B m)-F_{t}(d B m)\right]-\mathrm{SNR}_{\text {min }}
\end{aligned}
$$

Where PIIP3 is the input 3rd order intercept point and Ft is the background noise of the system. In this system, the value of IIP3 is $10 \mathrm{dBm}$, Ft is $-128 \mathrm{dBm}$ through the testing result of Noise figure meter. So, the SFDR of the system is $92 \mathrm{~dB}$. In the testing results, the maxim input signal could be $0 \mathrm{dBm}$. So, the SFDR of the system is more than $90 \mathrm{~dB}$.

The performance of the receiver can be evaluated by the bit error rate (BER) and selectivity of the detecting results.Additive white Gaussian noise channel (AWGN) is considered in the system, the probability of BER Pe is given by:

$$
P_{e}=\frac{1}{2} \exp \left(\frac{-r}{2}\right)
$$

Where $r=E_{b} / N_{0}, E_{b}$ is energy per bit, $N_{0}$ is the single-sided thermal noise spectral density. The relation between signal-to-noise ratio (SNR) and $\mathrm{Eb} / \mathrm{N} 0$ is given by 
$\frac{E_{b}}{N_{0}}=\frac{S T_{b}}{N / B}=\left(\frac{S}{N}\right)\left(\frac{B}{R}\right)=(S N R)\left(\frac{B}{R}\right)$

with SNR known, formula (10) can be expressed as [10]:

$$
P_{e}=\frac{1}{2} \exp \left(-\frac{P_{i n, \min }}{2 K\left[T_{a}+(F-1) T_{0}\right]} \frac{1}{R}\right.
$$

\section{EXPERIMENTAL}

In order to verify the performance of the receiver, we test it use real signal in the world.

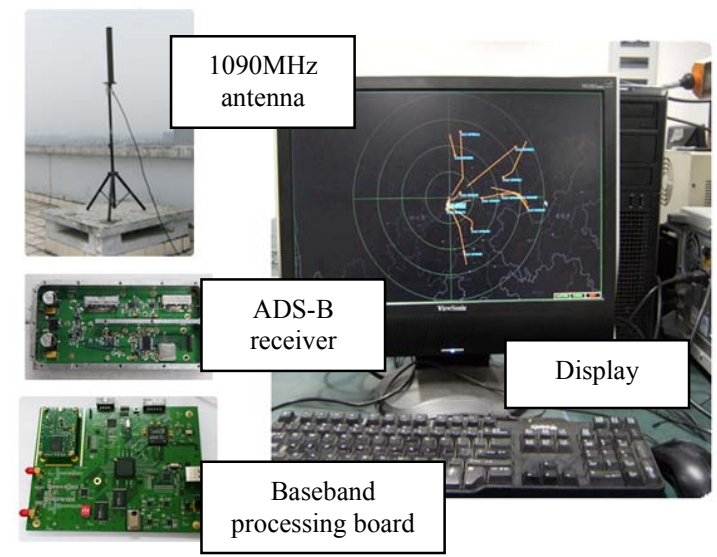

Figure 4. ADS-B station diagram

Fig.4 shows the block diagram of the testing environment ADS-B signals will be received and detected by the receiver, which will immediately transmit them to the baseband processing board. The baseband processing board analyzes this information and then transfers the decoded ADS-B data to the controllers' screens at the nearest air traffic control center.
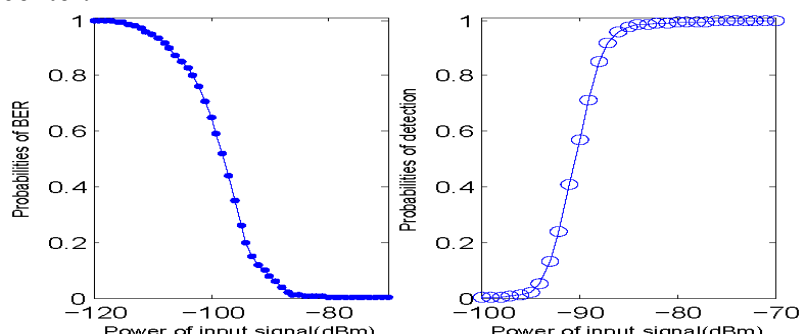

Figure 5. Probabilities of Detection and BER V.S. power of input signal

Figure 5 shows the Probabilities of bit error rate(BER) and detection with different input signal's power, which is done by using the analog signal generator. Probabilities of BER (PBER) are represented on part (a), while the Probabilities of detection $(\mathrm{Pd})$ are represented on the right. It is concluded that PBER decreases but Pd increases along with rise of the signals' power. The probabilities of detection are almost $80 \%$ when the power of the input signal is -
$90 \mathrm{dBm}$, and is almost $100 \%$ when the power is $-82 \mathrm{dBm}$. The performance of the receiver meets the requirements.

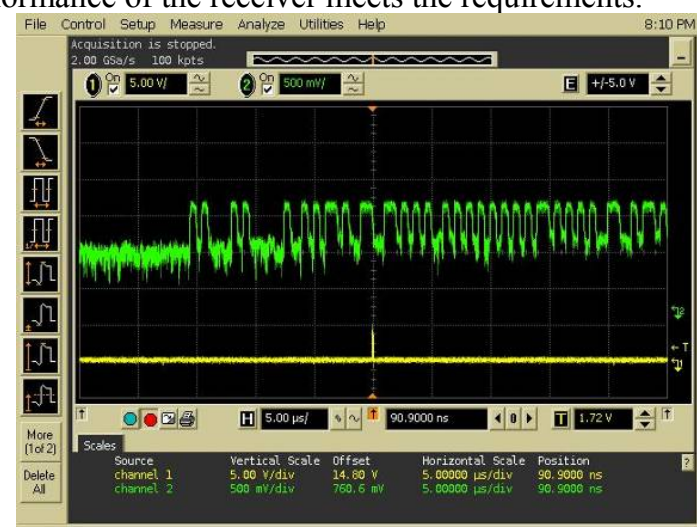

Figure 6. The detecting result from real ADS-B signal

Fig.6 shows the test result from real ADS-B signal. The green curve is the logarithmic detecting result of the RF receiver, and the yellow one is the detecting and decoding result from the baseband processing board. Only when the baseband board could decode the signal, the signal is considered as a surefire result from the RF module. The receiver could detect as far as $370 \mathrm{~km}$ from it by analyzing the decoding result of the receiving ADS-B signal.

\section{SUMMARIES}

This paper presents a high-sensitivity ADS-B receiver scheme, which is totally different from the traditional Superheterodyne ADS-B receiver. The sensitivity, noise figure, spurious-free dynamic range and bit error rate of the receiver are analyzed in detail. Through the testing result, the performance of the receiver is satisfactory.

\section{REFERENCES}

[1] C. Rekkas, and M. Rees,: "Towards ADS-B implementation in Europe," Digital Communications - Enhanced Surveillance of Aircraft and Vehicles, 2008. TIWDC/ESAV 2008. Tyrrhenian International Workshop on, PP:1-4

[2] Federal Aviation Administration, "Automatic Dependent Surveillance- Broadcast (ADS-B) Out Performance Requirements To Support Air Traffic Control (ATC) Service; Final Rule,"May, 2010

[3] E. Perl, "Review of airport surface movement radar technology", Radar, 2006 IEEE Conference on, April 2006,

[4] K. Sampigethaya, and R. Poovendran, "Validation techniques for ADS-B surveillance data," Proc. IEEE/AIAA 21th, Digital Avionics Systems Conference (DASC), Oct.2002, PP:3E2-1

[5] Y. Yorozu, M. Hirano, K. Oka, and Y. Tagawa, "Electron spectroscopy studies on magneto-optical media and plastic substrate interface," IEEE Transl. J. Magn. Japan, vol. 2, pp. 740-741, August 1987 [Digests 9th Annual Conf. Magnetics Japan, PP: 301, 1982].

[6] A. Helmy, K. Sharaf, and H. Raga, "On the SFDR performance of BJT RF circuits, an analytical approach.", Microelectronics, IEEE/ICM 12th.PP:375. Oct 2000

[7] Kan Baoqiang, Cai Li, Zhu Hongsong and Xu Yongjun, "Accurate energy model for WSN node and its optimal design," Journal Of Systems Engineering And Electronics, vol.19,No.3,2008,PP:427-433 